\title{
Hopf Bifurcation Control in a FAST TCP and RED Model via Multiple Control Schemes
}

\author{
Dawei Ding,,2 Chun Wang, ${ }^{1}$ Lianghui Ding, ${ }^{3}$ Nian Wang, and Dong Liang ${ }^{1}$ \\ ${ }^{1}$ School of Electronics and Information Engineering, Anhui University, Hefei, Anhui 230601, China \\ ${ }^{2}$ Ira A. Fulton Schools of Engineering, Arizona State University, Tempe, AZ 85287, USA \\ ${ }^{3}$ School of Electronic Information and Electrical Engineering, Shanghai Jiao Tong University, Shanghai 200240, China
}

Correspondence should be addressed to Lianghui Ding; lhding@sjtu.edu.cn

Received 10 November 2015; Accepted 5 May 2016

Academic Editor: Petko Petkov

Copyright (C) 2016 Dawei Ding et al. This is an open access article distributed under the Creative Commons Attribution License, which permits unrestricted use, distribution, and reproduction in any medium, provided the original work is properly cited.

\begin{abstract}
We focus on the Hopf bifurcation control problem of a FAST TCP model with RED gateway. The system gain parameter is chosen as the bifurcation parameter, and the stable region and stability condition of the congestion control model are given by use of the linear stability analysis. When the system gain passes through a critical value, the system loses the stability and Hopf bifurcation occurs. Considering the negative influence caused by Hopf bifurcation, we apply state feedback controller, hybrid controller, and time-delay feedback controller to postpone the onset of undesirable Hopf bifurcation. Numerical simulations show that the hybrid controller is the most sensitive method to delay the Hopf bifurcation with identical parameter conditions. However, nonlinear state feedback control and time-delay feedback control schemes have larger control parameter range in the Internet congestion control system with FAST TCP and RED gateway. Therefore, we can choose proper control method based on practical situation including unknown conditions or parameter requirements. This paper plays an important role in setting guiding system parameters for controlling the FAST TCP and RED model.
\end{abstract}

\section{Introduction}

The number of Internet users and the volume of contents through have grown significantly in recent years. Imbalance between fast-growing transport demand and limited network supply has resulted in severe congestion in many transport networks [1]. Traditional TCP protocols, for example, TCP New Reno and TCP Tahoe, use slow start, congestion avoidance, and fast retransmit algorithms [2] to adjust the source rate based on simple window based congestion control. The active queue management (AQM) schemes are implemented in the routers of communication networks to react to incipient congestion before the queue overflows [3]. Therefore, the best treatment of the congestion occurs when TCP and AQM algorithms work together [4].

Traditional congestion algorithms do not have large bandwidth-delay products for high speed networks. This has motivated several congestion control algorithms of high speed system, including HSTCP, STCP, FAST TCP, and BIC
TCP [5]. FAST TCP is a congestion control algorithm for high speed networks with large bandwidth-delay products and uses the queuing delay as congestion measure, which enables FAST TCP to detect congestion without packet loss [6]. Many research efforts have been devoted to the analysis of the stability property of FAST TCP. In [7], inherently inputto-state stability as well as globally asymptomatic stability without any specific conditions on the tuning parameter $\alpha$ or update gain $\gamma$ for FAST TCP operation has been proved by Razumikhin-type nonlinear small gain theorem. In [8-10], authors show sufficient conditions for the global asymptotic stability of FAST TCP in a single-link single-source network and multiple-link network with feedback delays. Then, stability criterion for double time-delay FAST TCP systems has proved the local asymptotical stability with the existence of delay when the round trip propagation delay is smaller than the queuing delay [11].

However, several nonlinear dynamical behaviors, such as periodic oscillatory behavior, chaos, and bifurcation, are 
harmful to system [12]. Thus, it is necessary to investigate a variety of control schemes to delay these undesired behaviors. In general, bifurcation control refers to the control of bifurcation properties of nonlinear dynamic system, thereby resulting in some desired output behaviors of the system [13]. The author in [14] has proposed a hybrid controller to delay the onset of the Hopf bifurcation in a wireless access network. And paper [13] has used a state feedback method to control the bifurcation for a novel congestion control model. In [15], an impulsive control strategy has been applied to the FAST TCP and RED model for controlling bifurcation. A nonlinear feedback controller has been designed to control the Hopf bifurcation behavior and the amplitude of limit cycle emerging from the modified Lorenz system in [16]. In this paper, three controllers, state feedback controller [13], hybrid controller [17, 18], and time-delay feedback controller [19-21], are applied to FAST TCP and RED model, respectively, for postponing the occurrence of Hopf bifurcation. The nonlinear state feedback controller can control the Hopf bifurcation to achieve desirable behaviors. It has advantage of not requiring any prior knowledge but the natural equilibrium point of the system. The time-delay feedback control which does not need the steady state of the congestion algorithm and changes the original algorithm is applied to the congestion control algorithm [16]. The hybrid control strategy can be applied to any component of a several-dimensional dynamical system and is still effective even when the system becomes chaotic [22]. Therefore, this paper compares the above three control strategies to determine an appropriate control algorithm which can achieve the best stability in FAST TCP and RED model.

The rest of the paper is organized as follows. Some results in uncontrolled system are concluded in Section 2. In Section 3, controlled systems with different control methods are introduced, and the existence of the Hopf bifurcation is proved by linear analysis. Section 4 gives some corresponding simulations to confirm the theoretical results. Finally, conclusion is demonstrated in Section 5.

\section{Hopf Bifurcation in Fast TCP and RED Model}

In this section, we show the main results of Hopf bifurcation for the FAST TCP model in [10]. The model can be described by the following nonlinear differential equations:

$$
\begin{aligned}
& \dot{w}(t)=\gamma\left(\frac{\alpha}{d+q(t)}-\frac{q(t)}{(d+q(t))^{2}} w(t)\right), \\
& \dot{p}(t)=\frac{1}{c}\left(\frac{w\left(t-\tau^{\mathrm{f}}\right)}{d+\left(p\left(t-R_{0}\right)\right)}-c\right),
\end{aligned}
$$

where $w(t)$ denotes congestion window of the source, $c$ is the transmission capacity, $p(t)$ is the queuing delay, and $q(t)=$ $p\left(t-\tau^{\mathrm{b}}\right)$ is the queuing delay observed by the source. The round trip time $R(t)=\tau^{\mathrm{f}}+\tau^{\mathrm{b}}$, where $\tau^{\mathrm{f}}$ is the forward delay from source to link and $\tau^{\mathrm{b}}$ is the backward delay in the feedback path from link to source, and $d$ represents the constant round trip propagation time defined as the minimum achievable round trip delay. Thus, $R(t)=d+p(t-$ $\left.\tau^{\mathrm{b}}\right)$. The parameter $\alpha(\alpha>0)$ is the number of the packets that each source attempts to maintain in the network buffers at equilibrium point; $\gamma$ is the source control parameter with $\gamma \in(0,1]$. The congestion window $w(t)$ and the queuing delay $p(t)$ are nonnegative.

Equation (1) can be written as follows with only one delay:

$$
\begin{aligned}
& \dot{w}_{1}(t) \\
& \quad=\gamma\left(\frac{\alpha}{d+p_{1}\left(t-R_{0}\right)}-\frac{p_{1}\left(t-R_{0}\right)}{\left(d+p_{1}\left(t-R_{0}\right)\right)^{2}} w_{1}(t)\right), \\
& \dot{p}_{1}(t)=\frac{1}{c}\left(\frac{w(t)}{d+\left(p_{1}\left(t-R_{0}\right)\right)}-c\right),
\end{aligned}
$$

and the equilibrium point is

$$
\begin{aligned}
& w_{0}=\frac{\alpha R_{0}}{\left(R_{0}-d\right)}, \\
& p_{0}=\frac{\alpha}{c}=R_{0}-d .
\end{aligned}
$$

Lemma 1 (see [1]). All roots of the characteristic equation $\lambda+$ $\mu+v e^{-\lambda}=0$, where $\mu$ and $v$ are real, have negative real parts if and only if (1) $\mu>1$ and $-\mu<v<\sqrt{\mu^{2}+\xi^{2}}$ where $\xi=$ $-\mu \tan \xi, 0<\xi<\pi$, if $\mu \neq 0$ and $\xi=\pi / 2$ if $\mu=0$.

Theorem 2 (see [10]). For system (2), one knows that the equilibrium point is asymptotically stable when $\alpha>\alpha_{0}$ and is unstable when $0<\alpha<\alpha_{0}$. In addition, system (2) exhibits a Hopf bifurcation when $\alpha=\alpha_{0}$, where

$$
\begin{gathered}
\gamma=\frac{w_{0}\left(w_{0}-\sin \left(w_{0}\right)\right)}{\cos \left(w_{0}\right)} \\
\alpha_{0}=\frac{c d\left(w_{0} \sin \left(w_{0}\right)-1\right)}{w_{0}^{2}-2 w_{0} \sin \left(w_{0}\right)+1},
\end{gathered}
$$

where $w_{0} \in(2 k \pi, 2 k \pi+\pi / 2), k=0,1,2, \ldots$.

\section{Hopf Bifurcation in Controlled System}

In this section, we add three control methods to the original model [10] for postponing the Hopf bifurcation in FAST TCP model of the Internet congestion control system.

First, we give state feedback control model:

$$
\begin{aligned}
\dot{w}(t)= & \gamma\left(\frac{\alpha}{d+p\left(t-R_{0}\right)}-\frac{p\left(t-R_{0}\right)}{\left(d+p\left(t-R_{0}\right)\right)^{2}} w(t)\right) \\
& -\frac{h_{1}}{R_{0}}\left(w(t)-w_{0}\right)-\frac{h_{2}}{R_{0}}\left(w(t)-w_{0}\right)^{2} \\
& -\frac{h_{3}}{R_{0}}\left(w(t)-w_{0}\right)^{3}, \\
\dot{p}(t)= & \frac{1}{c}\left(\frac{w(t)}{d+p\left(t-R_{0}\right)}-c\right),
\end{aligned}
$$



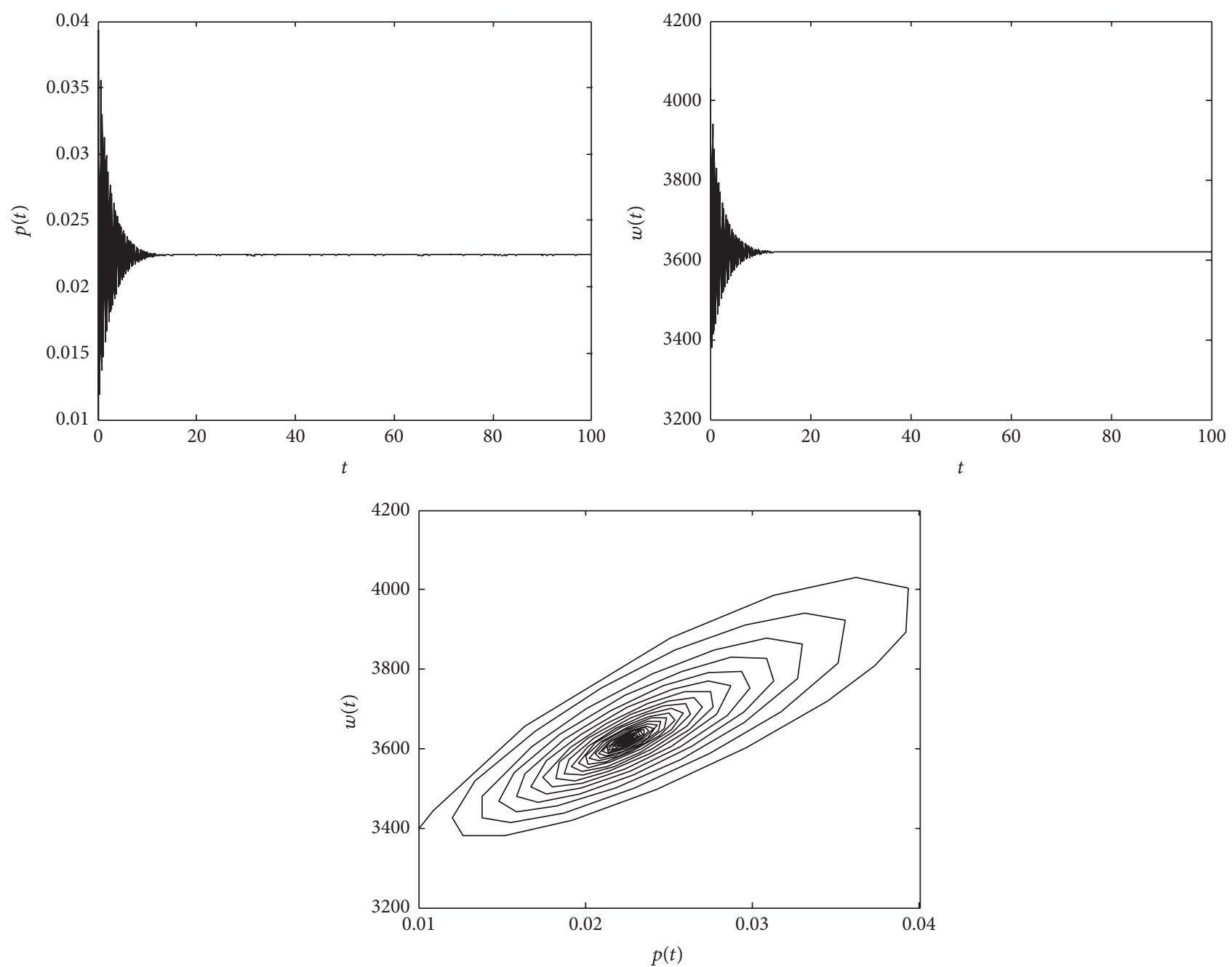

FIGURE 1: State plot and phase plot of uncontrolled system (2) with $\alpha=1120$.

hybrid control model:

$$
\begin{aligned}
\dot{w}(t)= & k \gamma\left(\frac{\alpha}{d+p\left(t-R_{0}\right)}-\frac{p\left(t-R_{0}\right)}{\left(d+p\left(t-R_{0}\right)\right)^{2}} w(t)\right) \\
& +(1-k)\left(w(t)-w_{0}\right), \\
\dot{p}(t)= & \frac{1}{c}\left(\frac{w(t)}{d+p\left(t-R_{0}\right)}-c\right),
\end{aligned}
$$

and time-delay control model:

$$
\begin{aligned}
\dot{w}(t)= & \gamma\left(\frac{\alpha}{d+p\left(t-R_{0}\right)}-\frac{p\left(t-R_{0}\right)}{\left(d+p\left(t-R_{0}\right)\right)^{2}} w(t)\right) \\
& -a\left(w(t)-w\left(t-R_{0}\right)\right), \\
\dot{p}(t)= & \frac{1}{c}\left(\frac{w(t)}{d+p\left(t-R_{0}\right)}-c\right),
\end{aligned}
$$

where $h_{1}, h_{2}$, and $h_{3}$ are negative feedback gain parameters, $k$ is the hybrid control parameter, and $a$ is time-delay feedback gain. The onset of Hopf bifurcation can be delayed by choosing appropriate parameters.

Secondly, we regard system (5) as an example for analysis. Note that the system equilibrium point is only related to $h_{1}$. It is clear that the controlled system has the same equilibrium point as the original system (2).

Let $y_{1}(t)=w-w_{0}$ and $y_{2}(t)=p-p_{0}$. Linearizing system (5) about the equilibrium point, we get

$$
\begin{aligned}
& \dot{y}_{1}(t)=\left(\frac{-\gamma p_{0}}{R_{0}^{2}}-\frac{h_{1}}{R_{0}}\right) y_{1}(t)-\frac{\gamma \alpha d}{p_{0} R_{0}^{2}} y_{2}\left(t-R_{0}\right), \\
& \dot{y}_{2}(t)=\frac{1}{R_{0} c} y_{1}(t)-\frac{1}{R_{0}} y_{2}\left(t-R_{0}\right),
\end{aligned}
$$

the characteristic equation of which is

$$
s^{2}+\left(\frac{\gamma p_{0}}{R_{0}^{2}}+\frac{h_{1}}{R_{0}}\right) s+\left(\frac{h_{1}}{R_{0}^{2}}+\frac{\gamma}{R_{0}^{2}}+\frac{s}{R_{0}}\right) e^{-s R_{0}}=0 .
$$



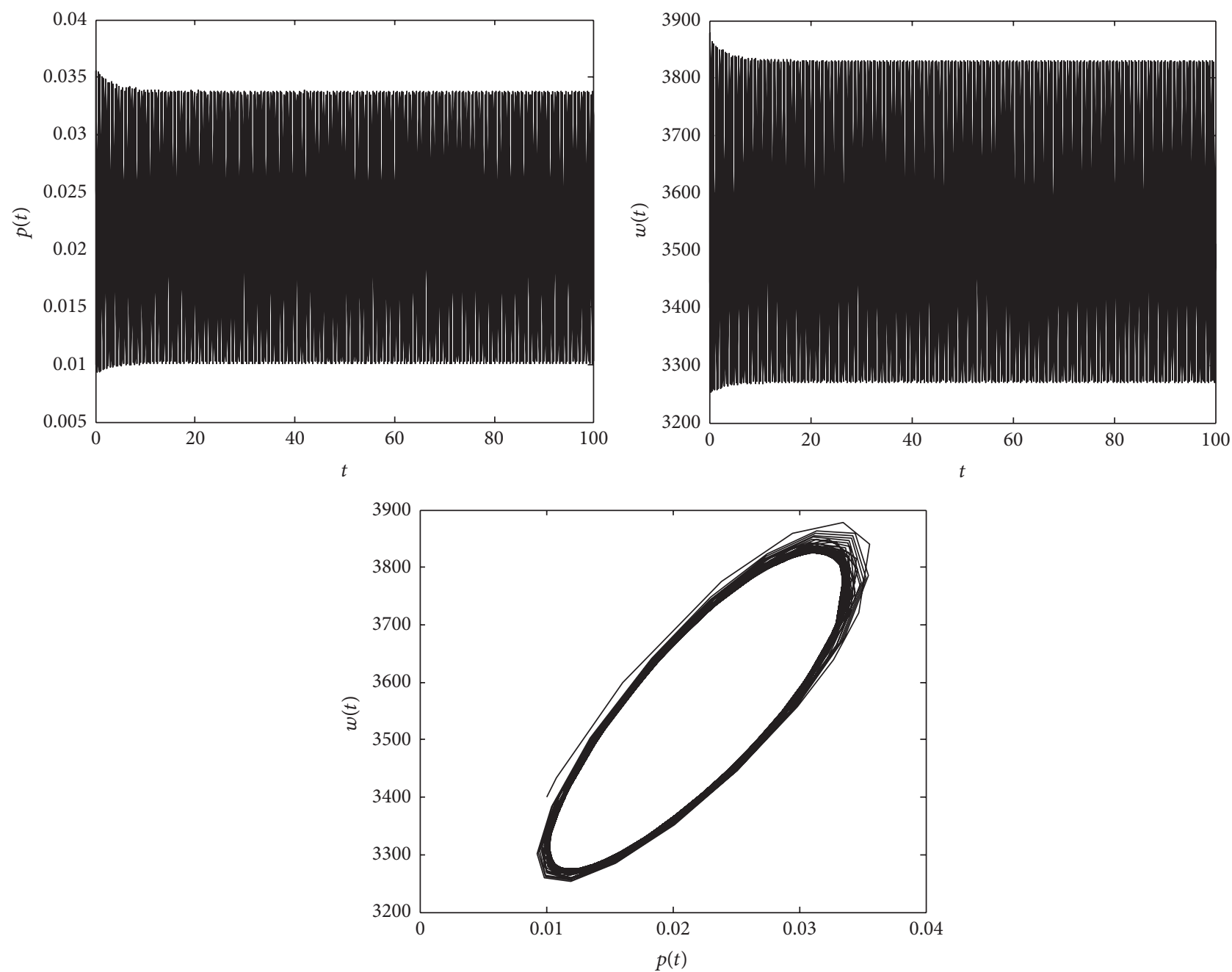

FIgURE 2: State plot and phase plot of uncontrolled system (2) with $\alpha=1000$.

Letting $\lambda=s R_{0}$, it becomes

$$
\lambda^{2}+\lambda e^{-\lambda}+\frac{\gamma \alpha}{\alpha+c d} \lambda+\gamma e^{-\lambda}+h_{1} \lambda+h_{1} e^{-\lambda}=0 .
$$

Similar to [10], let $\alpha \rightarrow+\infty$ and we get

$$
\left(\lambda+\gamma+h_{1}\right)\left(\lambda+e^{-\lambda}\right)=0 .
$$

So, we can be sure that $\lambda=-\gamma+h_{1}<0$, or

$$
\lambda+e^{-\lambda}=0
$$

According to Lemma 1, we know that the real parts in all the roots of (11) are negative. In this case, the equilibrium point is asymptotically stable. Then, when the value of $\alpha$ from $+\infty \rightarrow 0$, the critical value $\alpha_{0}$ appears. And an imaginary root $\lambda=i w_{0}\left(w_{0}>0\right)$ would occur for $\alpha=\alpha_{0}$.

Then, we can easily obtain

$$
\begin{aligned}
& \frac{d \lambda}{d \alpha} \\
& =\frac{-\gamma c d \lambda}{(\alpha+c d)^{2}\left[2 \lambda+\left(1-\lambda-\gamma+h_{1}\right) e^{-\lambda}+h_{1}+\gamma \alpha /(\alpha+c d)\right]} .
\end{aligned}
$$

From (10), we have

$$
e^{-\lambda}=\frac{\lambda\left(-h_{1}-\lambda\right)(\alpha+c d)-\gamma \alpha \lambda}{(\alpha+c d)\left(\lambda+\gamma+h_{1}\right)} .
$$

So, (13) can be written as

$$
\frac{d \lambda}{d \alpha}=\frac{-\gamma c d \lambda\left(\lambda+a \gamma+h_{1}\right) /(\alpha+c d)}{(\alpha+c d)\left[\lambda^{3}+\lambda^{2}+\left(\gamma+2 h_{1}\right) \lambda^{2}+(\gamma+h)\left(2-h_{1}\right) \lambda+\gamma \alpha\left(\lambda^{2}+\left(\lambda+h_{1}\right) \lambda+\gamma+h\right)+h_{1}\left(\gamma+h_{1}\right)\right]} .
$$



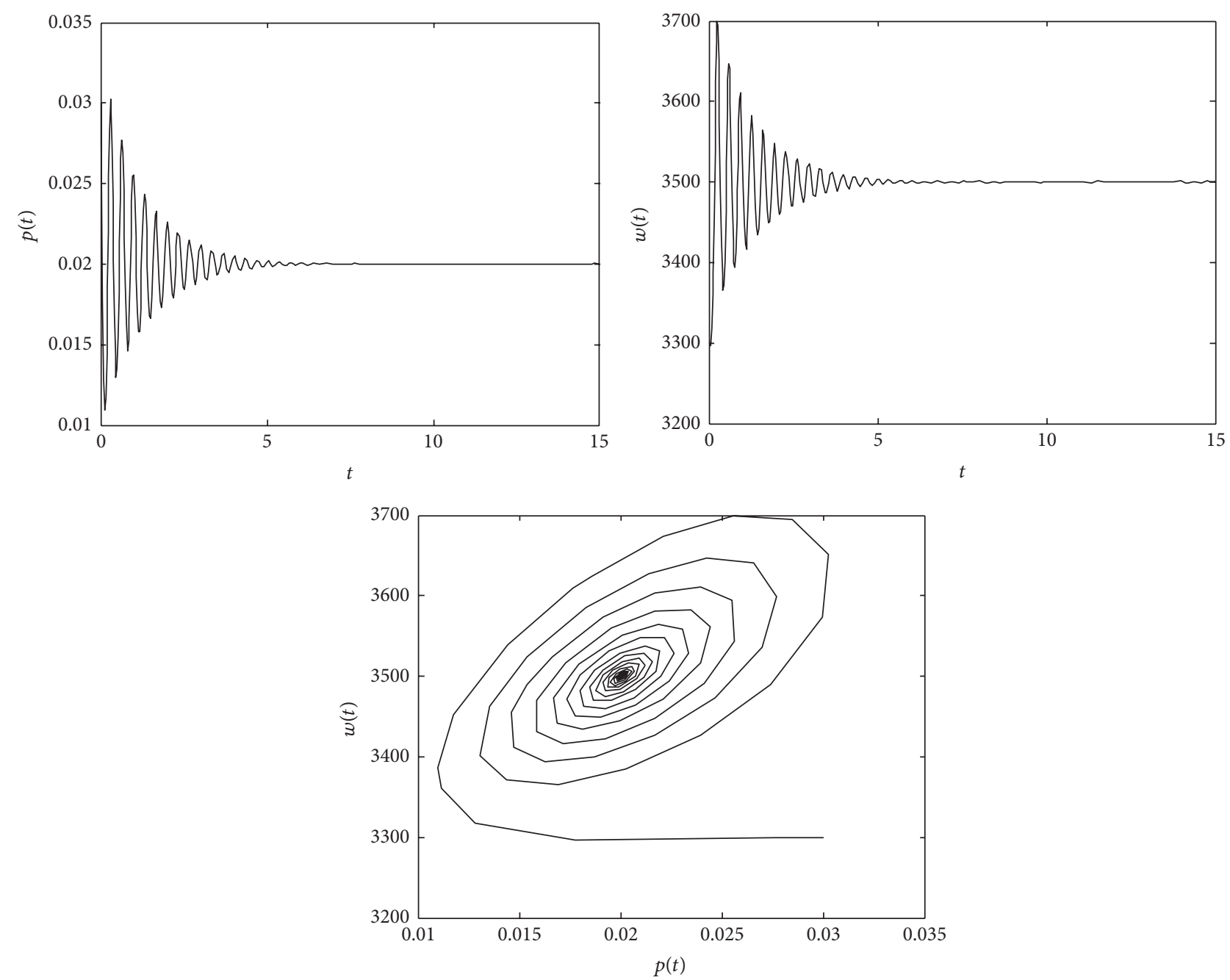

FIgURE 3: State plot and phase plot of state feedback controlled system (5) with $\alpha=1000$ and $h_{1}=0.8, h_{2}=0$, and $h_{3}=0$.

Substituting $\lambda=i w_{0}(w>0)$ into above equation, we have

$$
\left.\frac{d \lambda}{d \alpha}\right|_{\lambda=i w_{o}} \leq 0
$$

When $\alpha<\alpha_{0}$, the root of (10) has positive real part. Therefore, we can get that system (5) is stable for $\alpha>\alpha_{0}$.

Substituting $\lambda=i w_{0}\left(w_{0}>0\right)$ into (10), we get

$$
\begin{aligned}
& \gamma \sin w_{0}=w_{0} \cos w_{0}+h_{1} w_{0}+h_{1} \sin w_{0}+\frac{\gamma \alpha_{0} w_{0}}{\alpha_{0}+c d}, \\
& \gamma \cos w_{0}=w_{0}^{2}-w_{0} \sin w_{0}+h_{1} \cos w_{0} .
\end{aligned}
$$

Obviously, we can know that

$$
\begin{gathered}
\alpha_{0}=\frac{c d\left(w_{0} \sin w_{0}-h_{1} \cos w_{0}-1\right)}{w_{0}^{2}-2 w_{0} \sin w_{0}+1}, \\
\gamma=\frac{w_{0}^{2}-w_{0} \sin w_{0}-h_{1} \cos w_{0}}{\cos w_{0}} .
\end{gathered}
$$

From (10),

$$
\begin{aligned}
\frac{d \Delta(\lambda, \alpha)}{d \lambda}= & 2 \lambda+e^{-\lambda}-\lambda e^{-\lambda}+\frac{\gamma \alpha}{\alpha+c d}-\gamma e^{-\lambda}+h_{1} \\
& -h_{1} e^{-\lambda} .
\end{aligned}
$$

So,

$$
\begin{aligned}
& \left.\frac{d \Delta(\lambda, \alpha)}{d \lambda}\right|_{\lambda=i w_{0}} \\
& =\left(\cos \left(w_{0}\right)-w_{0}^{2}+\frac{\gamma \alpha_{0}}{\alpha_{0}+c d}-h_{1} \cos \left(w_{0}\right)+h_{1}\right) \\
& \quad+i\left(2 w_{0}-\sin \left(w_{0}\right)+\frac{\gamma \alpha_{0} w_{0}}{\alpha_{0}+c d}+h_{1} \sin \left(w_{0}\right)\right)
\end{aligned}
$$

$\neq 0$.

Following [10] and the above equations, some results hold:

(1) When $\alpha=\alpha_{0}$, an imaginary root $\lambda=i w_{0}\left(w_{0}>\right.$ 0 ) would occur and other roots of (10) have strictly 

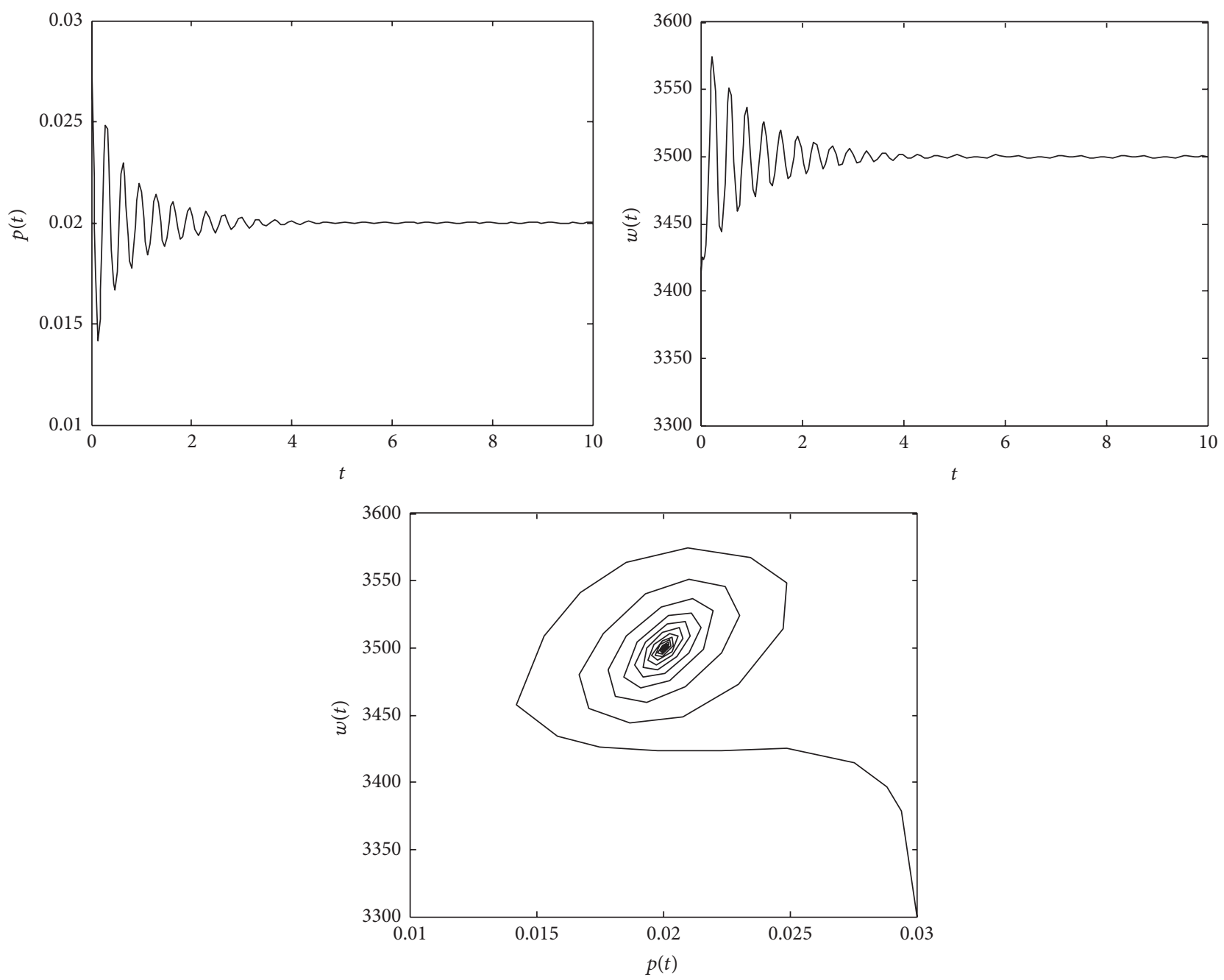

FIGURE 4: State plot and phase plot of state feedback controlled system (6) with $\alpha=1000$ and $h_{1}=0.8, h_{2}=0.0003$, and $h_{3}=0.0003$.

negative real parts. And the transversality condition holds as well.

(2) When $\alpha<\alpha_{0}$, the linearized system has unstable roots with positive real parts.

Theorem 3. The equilibrium point of system (5) is asymptotically stable when $\alpha>\alpha_{0}$ and unstable when $0<\alpha<\alpha_{0}$. When $\alpha=\alpha_{0}$, it exhibits a Hopf bifurcation.

is

The Taylor expansion of (5) about the equilibrium point

$$
\begin{aligned}
\dot{y}_{1}(t)= & c_{1} y_{1}(t)+c_{2} y_{2}\left(t-R_{0}\right)+c_{3} y_{1}(t) y_{2}\left(t-R_{0}\right) \\
& +c_{4} y_{2}^{2}\left(t-R_{0}\right)+c_{5} y_{1}(t) y_{2}^{2}\left(t-R_{0}\right) \\
& +c_{6} y_{2}^{3}\left(t-R_{0}\right)-\frac{h_{2}}{R_{0}} y_{1}^{2}(t)-\frac{h_{3}}{R_{0}} y_{1}^{3}(t) \\
& -\cdots
\end{aligned}
$$

$$
\begin{aligned}
\dot{y}_{2}(t)= & c_{7} y_{1}(t)+c_{8} y_{2}\left(t-R_{0}\right)+c_{9} y_{1}(t) y_{2}\left(t-R_{0}\right) \\
& +c_{10} y_{2}^{2}\left(t-R_{0}\right)+c_{11} y_{1}(t) y_{2}^{2}\left(t-R_{0}\right) \\
& +c_{12} y_{2}^{3}\left(t-R_{0}\right)+\cdots,
\end{aligned}
$$

where $c_{1}=-\gamma \alpha / c R_{0}^{2}-h_{1} / R_{0}, c_{2}=-\gamma c d / R_{0}^{2}, c_{3}=\gamma(\alpha-$ $c d) / c R_{0}^{3}, c_{4}=2 \gamma c d / R_{0}^{3}, c_{5}=\gamma(2 c d-\alpha) / c R_{0}^{4}, c_{6}=-3 \gamma c d / R_{0}^{4}$, $c_{7}=1 / c R_{0}, c_{8}=-1 / R_{0}, c_{9}=-1 / c R_{0}^{2}, c_{10}=1 / R_{0}^{2}, c_{11}=1 / c R_{0}^{3}$, and $c_{12}=-1 / R_{0}^{3}$.

Then, let $\alpha=\alpha_{0}+\mu, y(t)=\left(y_{1}(t), y_{2}(t)\right)^{T}$, and $y_{t}(\theta)=$ $y(t+\theta)$ for $\theta \in\left[-R_{0}, 0\right]$; then the Hopf bifurcation value of (12) is $\mu=0$. For initial condition $\varphi(\theta)=\left(\varphi_{1}(\theta), \varphi_{2}(\theta)\right)^{T} \epsilon$ $C\left[-R_{0}, 0\right]$. Equation (21) can be written as

$$
\begin{aligned}
& \dot{y}(t)=L_{\mu}\left(y_{t}\right)+F\left(y_{t}, \mu\right), \\
& L_{\mu} \varphi=B_{1} \varphi(0)+B_{2} \varphi\left(-R_{0}\right),
\end{aligned}
$$

where $B_{1}=\left(\begin{array}{ll}c_{1} & 0 \\ c_{7} & 0\end{array}\right)$ and $B_{2}=\left(\begin{array}{ll}0 & c_{2} \\ 0 & c_{8}\end{array}\right)$. 

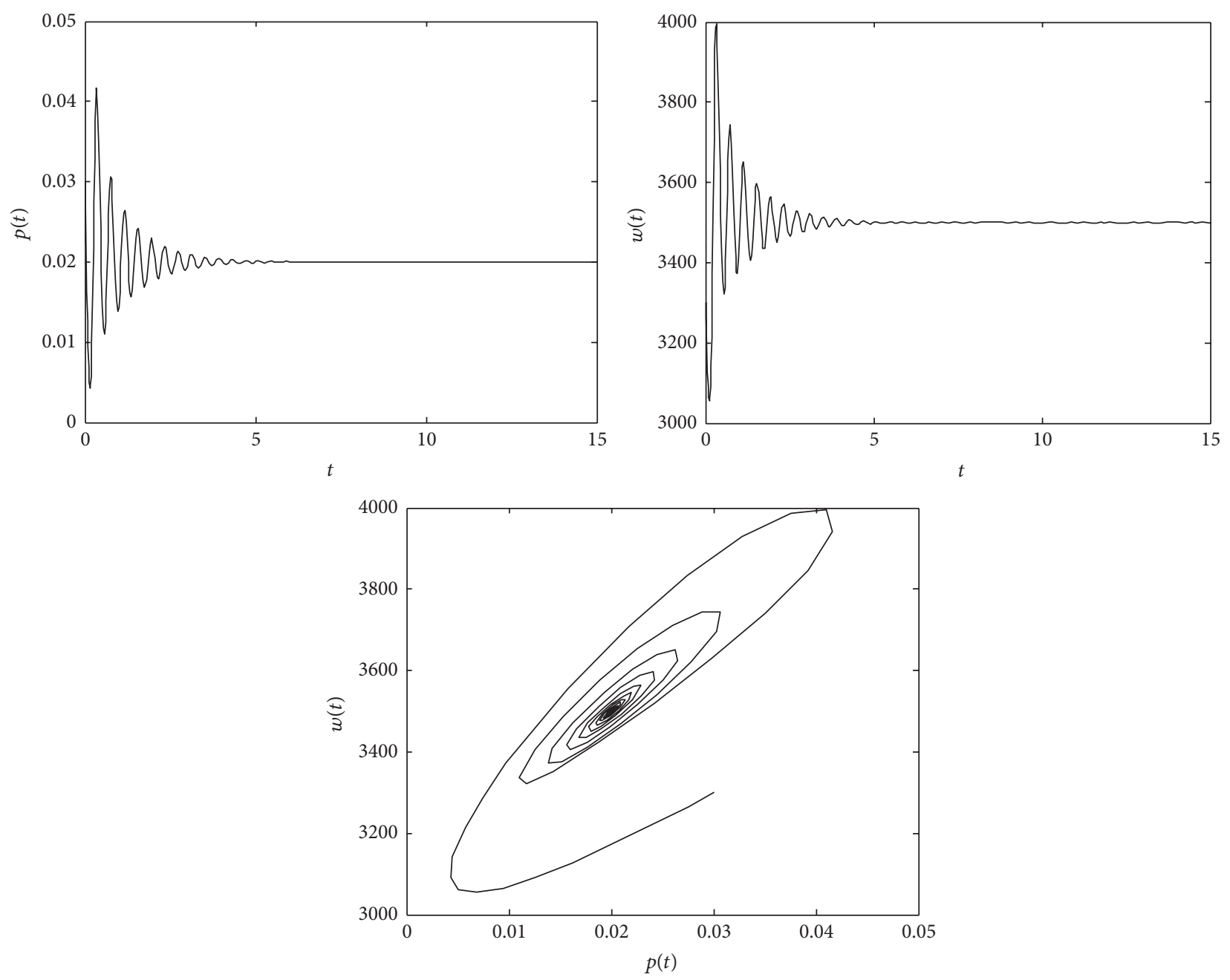

FIGURE 5: State plot and phase plot of hybrid controlled system (5) with $\alpha=1000$ and $k=0.8$.

According to the normal form theorem and center manifold theory, we have the following theorem based on the conclusion in [10].

Theorem 4. For system (5), when $\alpha=\alpha_{0}$, the following results hold:

(1) $\mu_{2}$ determines the direction of the Hopf bifurcation. If $\mu_{2}>0(<0)$, the Hopf bifurcation is supercritical (subcritical) and the bifurcation periodic solutions exist for $\alpha>\alpha_{0}\left(\alpha<\alpha_{0}\right)$.

(2) $\beta_{2}$ determines the stability of the bifurcating periodic solution. If $\beta_{2}<0(>0)$, the bifurcating periodic solutions are stable (unstable).

(3) $T_{2}$ determines the Hopf bifurcation of the bifurcation periodic solution. If $T_{2}>0(<0)$, the period increases (decreases),

where

$$
\begin{aligned}
C_{1}(0) & =\frac{i}{2 \omega_{0}}\left(g_{20} g_{11}-2\left|g_{11}\right|^{2}-\frac{1}{3}\left|g_{02}\right|^{2}\right)+\frac{g_{21}}{2}, \\
\mu_{2} & =-\frac{\operatorname{Re}\left\{C_{1}(0)\right\}}{\operatorname{Re} \lambda^{\prime}(0)},
\end{aligned}
$$

$$
\begin{aligned}
& T_{2}=-\frac{\operatorname{Im}\left\{C_{1}(0)\right\}+\mu_{2} \operatorname{Im} \lambda^{\prime}(0)}{\omega_{0}}, \\
& \beta_{2}=2 \operatorname{Re}\left\{C_{1}(0)\right\},
\end{aligned}
$$

where $C_{1}(0)$ is the Lyapunov coefficient. In general, the parameters $h_{2}, h_{3}$ as well as $h_{1}$ can be used to tune the above parameter which decide direction and stability of Hopf bifurcation.

\section{Numerical Simulation Examples}

In this section, we present numerical simulation to verify the analytic results and the control effects of three algorithms. We use the same parameters as those in [10]: $r=0.8 ; c=50000$ packets/s; and $d=0.05$; then we have $\alpha_{0}=1118$ from (4). From Theorem 4, we get $C_{1}(0)=-0.0115-i 0.0090, \mu_{2}=$ $-0.0116<0$ (the bifurcation is subcritical), $\beta_{2}=-0.023<0$ (the periodic solutions are stable), and $T_{2}=0.0187>0$ (the period increases). Figure 1 shows that trajectories converge to the equilibrium point for $\alpha=1120>\alpha_{0}=1118$. When it is decreased to $\alpha=1000$ in system (5), the system loses stability and Hopf bifurcation occurs (see Figure 2). In other 

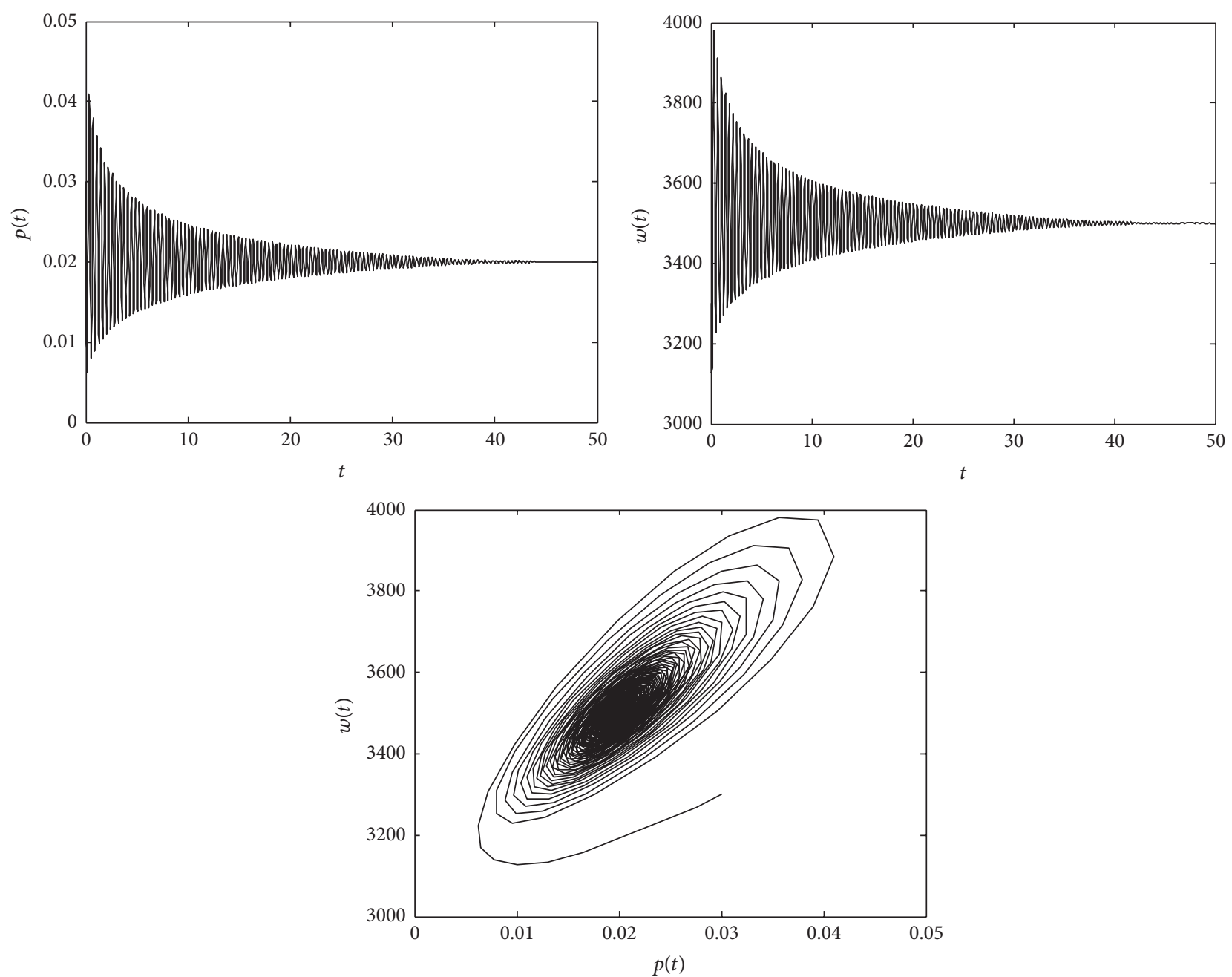

Figure 6: State plot and phase plot of time-delay feedback controlled system (6) with $\alpha=1000$ and $a=0.8$.

words, these figures verify the correctness of the theoretical analysis; that is, bifurcation periodic solution exists for the system value of $\alpha$ is slightly less than the critical value $\alpha_{0}$, and the bifurcation periodic solutions are stable.

Now, an appropriate control parameter can be chosen for controlling the Hopf bifurcation. For the purpose of comparing the effects of the three control methods, we use the same control parameter. When $h_{1}=0.8$, we see that the state feedback control model converges to the equilibrium point and the onset of the Hopf bifurcation is delayed (see Figure 3). Furthermore, the direction and period of Hopf bifurcation in system (4) are changed when $h_{1}=0.8, h_{2}=0.0003$, and $h_{3}=0.0003$ (see Figure 4).

From Theorem 4, we get

$$
\begin{aligned}
C_{1}(0) & =-0.0335-i 0.0043, \\
\mu_{2} & =-0.0128, \\
\beta_{2} & =-0.024, \\
T_{2} & =0.0197 .
\end{aligned}
$$

Figures 5 and 6 show that the system is asymptotically stable with hybrid and time-delay feedback control schemes instead of having a Hopf bifurcation as $\alpha=1000$. If $\alpha=1000$, we can compute $R_{0}=0.07, w_{0}=3500$, and $P_{0}=0.02$. From (18) we obtain $\alpha_{0}=423$ of system (5) with state feedback controller for $h_{1}=0.8, h_{2}=0, h_{3}=0$, and $\alpha_{0}=382$ of system (6) with hybrid controller for $k=0.8$ and $\alpha_{0}=960$ of system (7) with time-delay feedback controller for $a=0.8$. Above all, those figures prove that the three schemes which we proposed are effective to extend the stable range in parameter and guarantee a stable sending rate for a large delay in FAST TCP model. Figure 7 is the relationship between $h_{1}, k, a$, and $\alpha_{0}$ with $\gamma=0.8$. From Figure 7, we can see that the critical value of hybrid controller is the most sensitive to the change of control parameter, and time-delay feedback controller is the least sensitive in fixed control parameter range. Meanwhile, optional control parameter range of hybrid controllers is very limited which is $0.5 \leq 1 /(\gamma+1)<k \leq 1$, and timedelay feedback controller as well as nonlinear state feedback controller is infinite. Therefore, we can choose proper method based on practical situation including unknown conditions or parameter requirements. 


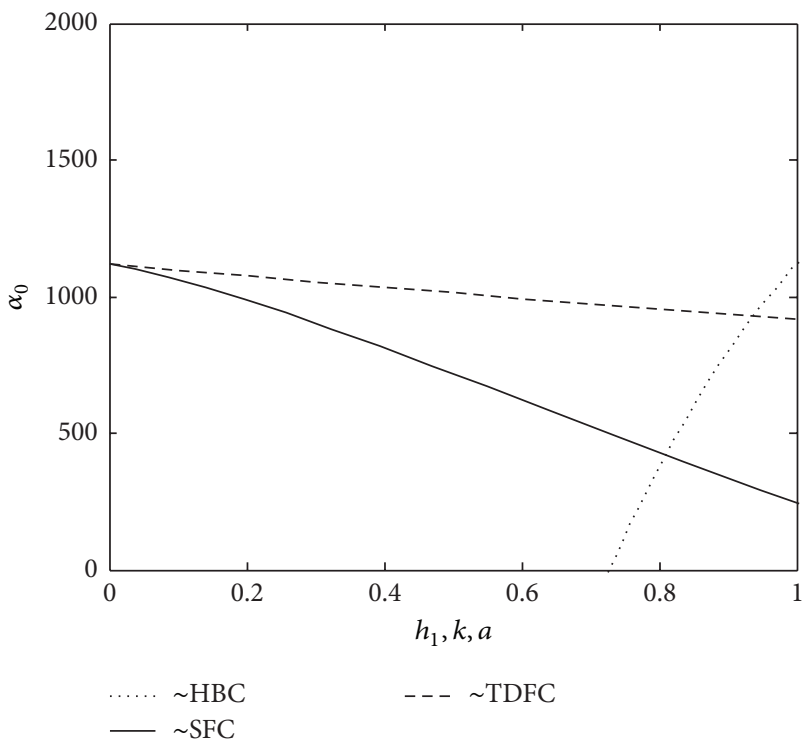

FIGURE 7: $\alpha_{0}$ dependence on $h_{1}, k, a$ with $\gamma=0.8$.

\section{Conclusion}

In this paper, state feedback, hybrid, and time-delay feedback controllers have been applied to the FAST TCP model in high speed network congestion control system. Among them, a system under a state feedback controller has been used to show the conditions for the local asymptotic stability. Then, we have represented simulation results in order to compare the control different effects between three control algorithms. As a result, they have effectively postponed the onset of the Hopf bifurcation that achieved desired dynamics behavior in FAST TCP model. By contrasting the three control schemes with identical parameter, we have known that hybrid controller is the most sensitive control strategy for FAST TCP and RED model. Besides, the control parameter interval of nonlinear state feedback and time-delay feedback control strategy is bigger than hybrid controller. Therefore, a method which meets some certain conditions is easily chosen, such as system dimension, prior knowledge, and parameter interval. The above analysis plays an important role in setting guiding system parameters for controlling the FAST TCP and RED model.

\section{Competing Interests}

The authors declare that they have no competing interests.

\section{Acknowledgments}

This paper is supported by the National Natural Science Foundation of China (no. 61201227), NSFC-Guangdong Joint Fund (no. U1201255), the Natural Science Foundation of Anhui Province (no. 1208085MF93), 211 Innovation Team of Anhui University (nos. KJTD007A and KJTD001B), and Chinese Scholarship Council. This paper is supported in part by NSFC China (Grants nos. 61221001, 61301117, and 61420106008), China Postdoctoral Science Foundation (Grant no. 2013M541519), and Shanghai Key Laboratory Funding (12DZ2272600).

\section{References}

[1] K. He, Z. Xu, and P. Wang, "A hybrid routing model for mitigating congestion in networks," Physica A: Statistical Mechanics and its Applications, vol. 431, pp. 1-17, 2015.

[2] M. Liu, H. Zhang, and L. Trajković, "Stroboscopic model and bifurcations in TCP/RED," in Proceedings of the IEEE International Symposium on Circuits and Systems (ISCAS '05), pp. 2060-2063, IEEE, Kobe, Japan, May 2005.

[3] D. Gu and W. Zhang, "Design of an $H_{\infty}$ based PI controller for AQM routers supporting TCP flows," in Proceedings of the 48th IEEE Conference on Decision and Control, 2009 held jointly with the 2009 28th Chinese Control Conference (CDC/CCC '09), pp. 603-608, IEEE, Shanghai, China, December 2009.

[4] S. Stancescu, A. A. Luca, and A. Caraivan, "TCP-AQM Algorithms which combat the computer networks' congestion," in Proceedings of the 22nd Telecommunications Forum Telfor (TELFOR '14), pp. 182-185, Belgrade, Serbia, November 2014.

[5] J. Wang, D. X. Wei, J.-Y. Choi, and S. H. Low, "Modelling and stability of FAST TCP," in Wireless Communications, vol. 143 of The IMA Volumes in Mathematics and Its Applications, pp. 331356, Springer, New York, NY, USA, 2007.

[6] D. X. Wei, C. Jin, S. H. Low, and S. Hegde, "FAST TCP: motivation, architecture, algorithms, performance," IEEE/ACM Transactions on Networking, vol. 14, no. 6, pp. 1246-1259, 2006.

[7] J.-Y. Choi, H. Kim, and S. Kwon, "Global asymptotic stability analysis of fast tcp using nonlinear small gain theorem," IEEE Communications Letters, vol. 18, no. 3, pp. 523-526, 2014.

[8] J.-Y. Choi, S.-Y. Kim, and J.-W. Kim, "Parameter condition for global asymptotic stability of FAST TCP in the presence of cross traffics," IEEE Communications Letters, vol. 14, no. 6, pp. 584586, 2010.

[9] F. Zhao, J. Zhou, N. Lu, and M. Zhang, "Stability analysis of FAST TCP based on Lyapunov function," in Proceedings of the 7th World Congress on Intelligent Control and Automation (WCICA '08), pp. 2136-2140, IEEE, Chongqing, China, June 2008.

[10] Z.-Q. Zhan, J. Zhu, and W. Li, "Stability and bifurcation analysis in a FAST TCP model with feedback delay," Nonlinear Dynamics, vol. 70, no. 1, pp. 255-267, 2012.

[11] H. Zhang and B. Liu, "Stability of FAST TCP with time-delay in single-link multi-source networks," in Proceedings of the IEEE International Conference on Information Networking and Automation (ICINA '10), pp. V2-259-V2-263, Kunming, China, October 2010.

[12] D. Ding, J. Zhu, X. Luo, and Y. Liu, "Delay induced Hopf bifurcation in a dual model of Internet congestion control algorithm," Nonlinear Analysis: Real World Applications, vol. 10, no. 5, pp. 2873-2883, 2009.

[13] M. Xiao, W. X. Zheng, and J. Cao, "Bifurcation control of a congestion control model via state feedback," International Journal of Bifurcation and Chaos in Applied Sciences and Engineering, vol. 23, no. 6, Article ID 1330018, 31 pages, 2013.

[14] D. Ding, X. Qin, T. Wu, N. Wang, and D. Liang, "Hopf bifurcation control of congestion control model in a wireless access network," Neurocomputing, vol. 144, pp. 159-168, 2014. 
[15] L. Feng, X. Gaoxiang, G. Zhi-Hong et al., "Stability analysis and control Hopf bifurcation in a FAST TCP model," in Proceedings of the 32nd IEEE Chinese Control Conference (CCC '13), pp. 1076-1080, Xian, China, July 2013.

[16] X. Wang, L. Deng, and W. Zhang, "Hopf bifurcation analysis and amplitude control of the modified Lorenz system," Applied Mathematics and Computation, vol. 225, no. 12, pp. 333-344, 2013.

[17] Z. Cheng and J. Cao, "Hybrid control of Hopf bifurcation in complex networks with delays," Neurocomputing, vol. 131, pp. 164-170, 2014.

[18] Z. Mao, H. Wang, D. Xu, and Z. Cui, "Bifurcation and hybrid control for a simple hopfield neural networks with delays," Mathematical Problems in Engineering, vol. 2013, Article ID 315367, 8 pages, 2013.

[19] S. Guo, G. Feng, X. Liao et al., "Hopf bifurcation control in a congestion control model via dynamic delayed feedback," Chaos, vol. 18, no. 4, pp. 1575-1629, 2009.

[20] L. Ma, D. Ding, T. Cao et al., "Bifurcation control in a small-world network model via TDFC," in Proceedings of the International Conference on Advances in Mechanical Engineering and Industrial Information, Atlantis Press, 2015.

[21] C.-L. Liu and Y.-P. Tian, "Eliminating oscillations in the Internet by time-delayed feedback control," Chaos, Solitons and Fractals, vol. 35, no. 5, pp. 878-887, 2008.

[22] Z. Liu and K. W. Chung, "Hybrid control of bifurcation in continuous nonlinear dynamical systems," International Journal of Bifurcation and Chaos in Applied Sciences and Engineering, vol. 15, no. 12, pp. 3895-3903, 2005. 


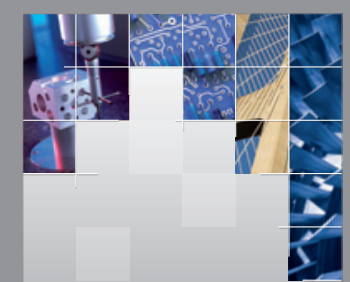

\section{Enfincering}
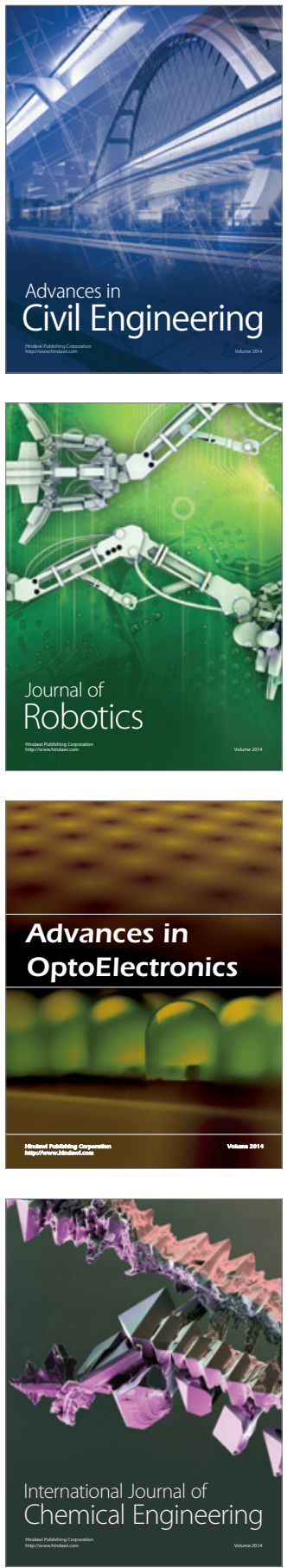

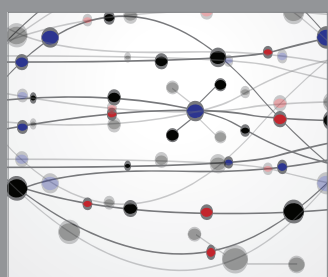

The Scientific World Journal

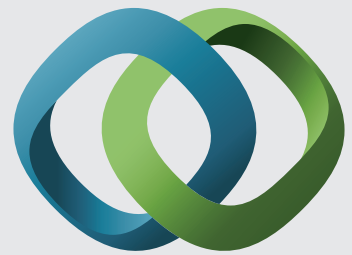

\section{Hindawi}

Submit your manuscripts at

http://www.hindawi.com
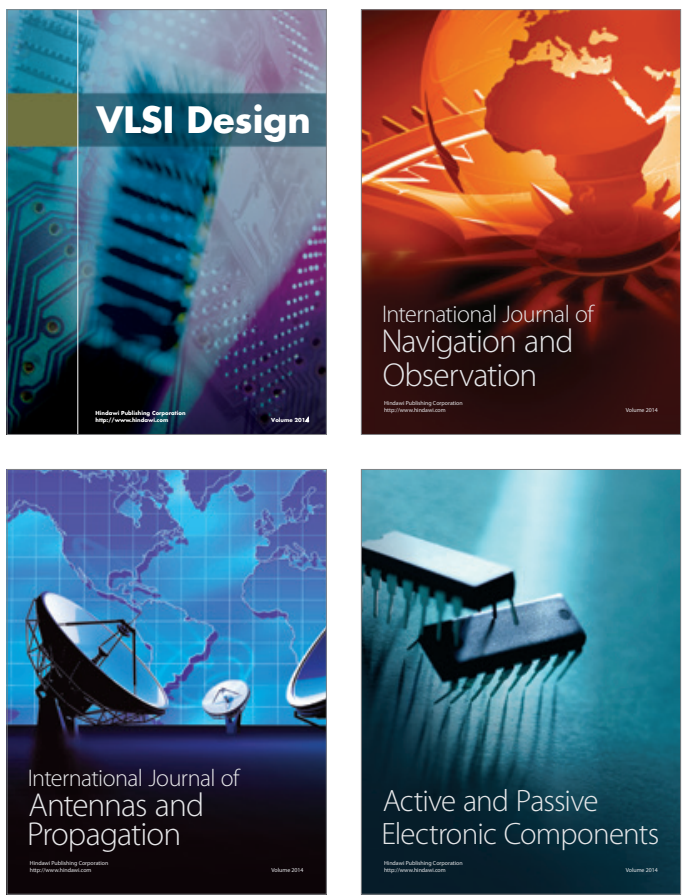
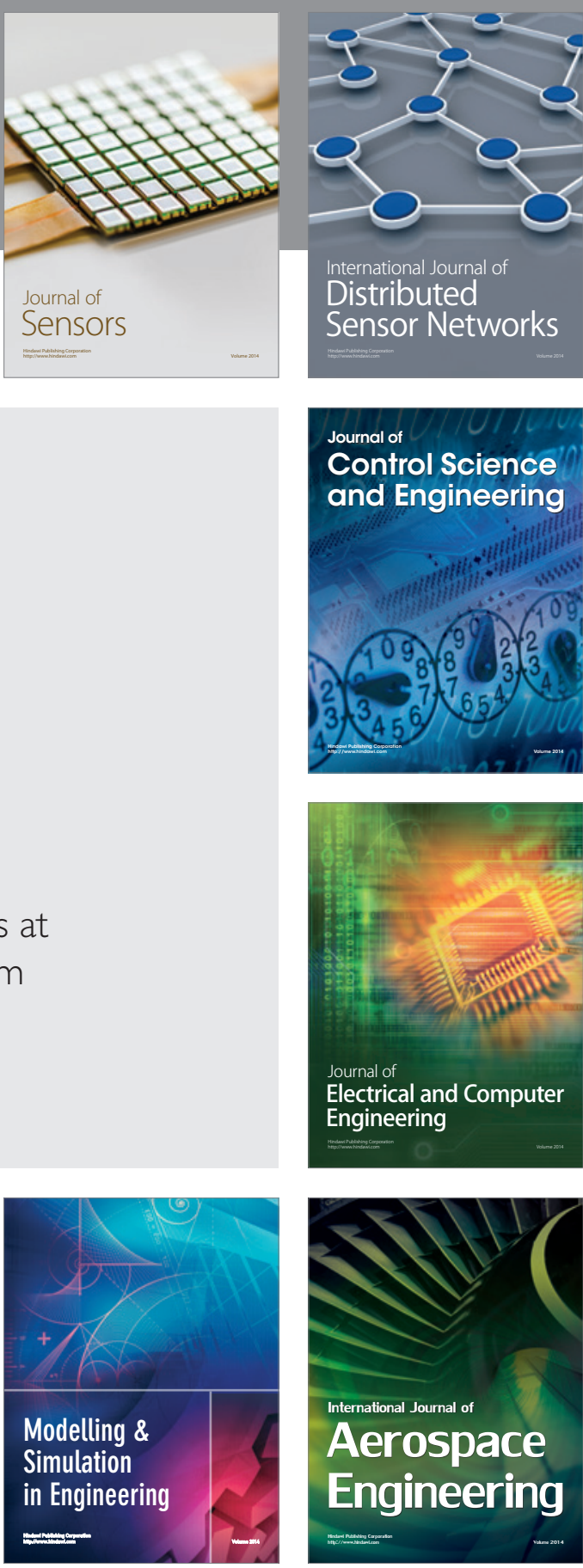

International Journal of

Distributed

Sensor Networks

Journal of

Control Science

and Engineering
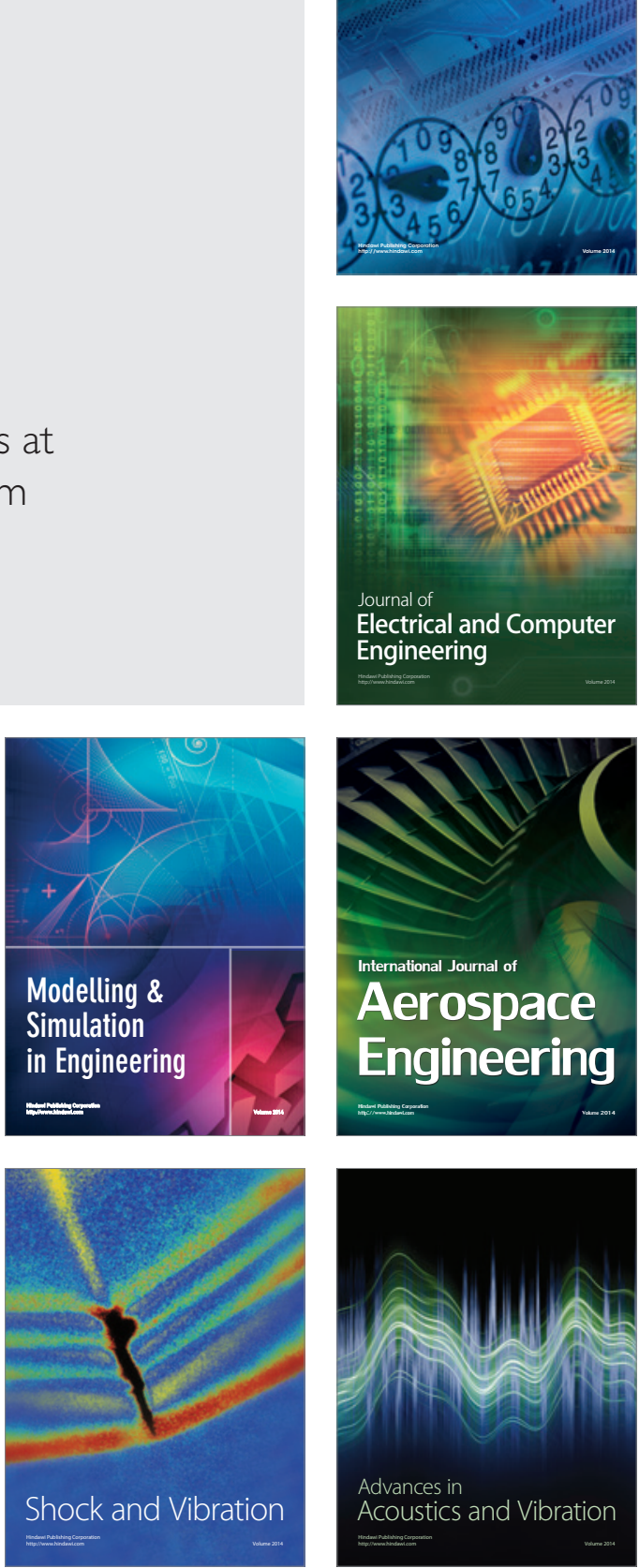